\title{
List of assessors
}

We would like to thank the following people who have acted as assessors of papers during the past year:

\begin{tabular}{|c|c|c|}
\hline D. Abrahamson & F. Hassanyeh & C. North \\
\hline C. Adams & K. Hawton & C. Nunn \\
\hline S. J. Aldridge & F. Holloway & M. R. Oates \\
\hline S. Bailey & D. S. Holman & A. Okasha \\
\hline D. Baldwin & J. Holmes & G. O'Sullivan \\
\hline C. Ballard & B. Hore & S. Onyett \\
\hline T. R. E. Barnes & A. O. House & O. A. Oyebode \\
\hline M. Basoglu & N. Hunt & W. Parry-Jones \\
\hline P. Bebbington & P. Huxley & E. Peck \\
\hline J. Bindman & M. Issacs & R. Persaud \\
\hline J. L. T. Birley & A. James & R. M. Philpott \\
\hline I. Blackburn & D. A. W. Johnson & J. S. Pippard \\
\hline C. V. R. Blacker & S. J. Johnston & B. Pitt \\
\hline R. Bloor & D. Jolley & I. Pullen \\
\hline C. Bools & P. Joseph & D. Rasitrick \\
\hline N. Bouras & O. Junaid & A. Reid \\
\hline S. Brandon & C. A. Kaplan & J. G. Rellly \\
\hline T. S. Brugha & C. Katona & G. Richardson \\
\hline T. Burns & R. Kemp & J. C. Rigby \\
\hline P. K. Carpenter & P. Kennedy & H. Ring \\
\hline P. R. Casey & D. Kingdon & B. Ritson \\
\hline J. Catalán & D. Kuipers & H. Rosenvinge \\
\hline J. Chick & R. Kumar & H. Rollin \\
\hline J. Coid & R. Laugharne & U. Schmidt \\
\hline J. Cookson & M. A. Launer & J. Scott \\
\hline D. Cottrell & P. Lelliott & E. Sein \\
\hline J. Crammer & J. Lindesay & T. Sensky \\
\hline F. Creed & R. Littlewood & E. C. Sheldrick \\
\hline K. A. Day & K. Lloyd & M. Shooter \\
\hline M. Donovan & A. Mackay & A. Sims \\
\hline R. Eastley & R. Macpherson & G. S. Stein \\
\hline N. Eastman & A. Maden & D. Storer \\
\hline T. Exworthy & S. Mann & F. Subotsky \\
\hline L. Fagin & S. Marriott & K. Sutherby \\
\hline T. Fahy & A. Masters & M. Swinton \\
\hline B. Ferguson & J. McCarthy & P. J. Taylor \\
\hline M. Field & R. G. McCreadie & C. Thompson \\
\hline C. Freeman & S. McIver & G. Thornicroft \\
\hline H. Freeman & K. McKenzie & P. Timms \\
\hline E. Garralda & S. Milne & L. Treliving \\
\hline D. Gasper & R. H. S. Mindham & P. Trigwell \\
\hline G. Glover & M. Mitcheson & S. Turner \\
\hline C. Godber & S. Moorey & C. Vassilas \\
\hline K. Gournay & H. G. Morgan & G. G. Wallis \\
\hline P. Graham & P. E. Mullen & J. P. Wattis \\
\hline A. Grounds & D. B. Mumford & M. Weller \\
\hline D. Grubin & E. Murphy & S. West \\
\hline J. Gunn & M. Murphy & F. Winkler \\
\hline E. Guthrie & D. H. Myers & A. E. Worrell \\
\hline M. Harper & H. Myles & T. Wykes \\
\hline J. Harris-Hendricks & D. A. Newby & A. Zigmond \\
\hline P. Harrison-Read & A. R. Nicol & \\
\hline
\end{tabular}

